\title{
Uma análise conjunta para identificação dos atributos de um dispositivo para reconhecimento de características de produtos alimentícios customizados
}

\section{A conjoint analysis to identify the attributes of a device to characteristics recognition in customized food products}

\author{
Luiz Philipi Calegari"* (D), Diego Castro Fettermann² (1) \\ ${ }^{1}$ Universidade Federal de Santa Catarina (UFSC), Engenharia de Alimentos e Engenharia de Produção, Laboratório \\ de Produtividade e Melhoria Contínua, Florianópolis/SC - Brasil. \\ ${ }^{2}$ Universidade Federal de Santa Catarina (UFSC), Engenharia de Produção, Florianópolis/SC - Brasil
}

${ }^{*}$ Corresponding Author: Luiz Philipi Calegari, Universidade Federal de Santa Catarina (UFSC), Engenharia de Alimentos e Engenharia de Produção, Rua Delfino Conto, s/n, Trindade, CEP: 88040-370, Florianópolis/SC - Brasil, e-mail: luizpcalegari@gmail.com

Cite as: Calegari, L. P., \& Fettermann, D. C. (2019). A conjoint analysis to identify the attributes of a device to characteristics recognition in customized food products. Brazilian Journal of Food Technology, 22, e2018024 https://doi.org/10.1590/1981-6723.02418

\begin{abstract}
Resumo
A possibilidade de combinação de ingredientes alimentícios em cada refeição torna complexo o reconhecimento das características dos alimentos personalizados. A utilização de dispositivos com tecnologias da Internet of Things (IoT) consiste em uma alternativa para os clientes acessarem informações dos alimentos personalizados. Nesta pesquisa, foram identificados cinco principais atributos na composição desses dispositivos com objetivo de reconhecimento dos ingredientes em refeições customizadas: (A) portabilidade, (B) precisão, (C) personalização de dieta, (D) análise de qualidade do produto alimentício e (E) preço. Este estudo possui como objetivo identificar a combinação desses atributos que geram valor em um dispositivo de reconhecimento de características alimentares de alimentos customizados. Foi realizado um planejamento fatorial fracionado $2^{5-1}$ para a apresentação dos atributos do dispositivo na forma de cenários, seguindo o método de análise conjunta baseada em escolha. Para a coleta de dados, foi realizada uma pesquisa com uma amostra de 303 respondentes. Ainda foram analisadas as variáveis moderadoras: gênero e presença de restrição alimentar. Os resultados obtidos apresentaram maior significância para os atributos: precisão de medida e análise de qualidade. Como contribuições, este estudo apresenta informações para o direcionamento de investimentos em pesquisa para elaboração de um dispositivo tecnológico com a finalidade de reconhecer características de um produto alimentício customizado em massa.
\end{abstract}

Palavras-chave: Personalização; Alimentos customizados; Customização em massa; Survey; Smart technology; loT; Restrição alimentar. 


\begin{abstract}
The possibility of combining food ingredients at each meal makes it difficult to recognize the characteristics of personalized foods. The use of devices with the Internet of Things (IOT) technologies is an alternative for customers to access customized food information. Five main attributes present in the composition of such devices, aiming ingredients recognition in customized meals, were considered in this study: (A) portability, (B) precision, (C) diet customization, (D) food quality control and (E) price. This study aims to identify the combination of such value generating attributes in a device of food characteristics recognition in customized foods. A fractional factorial design $2^{5-1}$ was used to display the device characteristics, in the form of scenarios, following the joint analysis method based on choice. For data collection, a survey was carried out with a sample of 303 respondents. Gender moderation and food restriction variables were also analyzed. The results present greater significance for the attributes of measurement accuracy and quality analysis. As contributions, this study presents information for investments targeting in research for the manufacturing of a technological device aiming the recognition of characteristics of a mass-modified food product.
\end{abstract}

Keywords: Personalization; Customized food; Mass customization; Survey; Smart technology; loT; Dietary restriction.

\title{
1 Introdução
}

As informações contidas nos rótulos dos alimentos consistem em uma importante fonte de informações para o cliente decidir sobre a compra do produto (Kriflik \& Yeatman, 2005). O efeito dos rótulos na decisão de compra está associado tanto a fatores relacionados com sua fabricação (Schuldt, 2013; Vasiljevic et al., 2015) quanto à disposição de informações sobre o produto alimentício (Kriflik \& Yeatman, 2005). A crescente necessidade de personalização dos produtos (Fettermann et al., 2017), inclusive alimentícios (Burton et al., 2009), indica uma disposição dos consumidores em pagar mais por alimentos que apresentem informações nutricionais mais específicas quando comparativamente àqueles com rótulos com informações nutricionais genéricas (Balcombe et al., 2016).

Com este propósito, são identificados aplicativos para smartphones que reconhecem as características nutricionais dos alimentos e a sua origem. Entre as tecnologias utilizadas nestes dispositivos, é possível identificar a utilização de etiquetas NFC (Near Field Communication) combinadas com sistemas RFID (Radio Frequency Identification), como no NUTRIFLECT (Reitberger et al., 2014) e o My Plate (Chen et al., 2014); sistemas de reconhecimento de código de barras (Arens-Volland et al., 2015); sistemas de espectrometria de cores (Coskun et al., 2013), scanners de espectrometria molecular (Arens-Volland et al., 2015; Makarona et al., 2016; Vanderroost et al., 2017); sistemas de reconhecimento de imagens (Meyers et al., 2015), e sistemas de reconhecimento de padrões de mastigação que permitem a entrada de voz para reconhecimento do alimento (Arens-Volland et al., 2015).

$\mathrm{Na}$ literatura, também podem ser identificados dispositivos com a finalidade de verificação da qualidade do produto alimentício. São utilizadas diferentes tecnologias para essa finalidade, tais como: espectrometria (Makarona et al., 2016; Vanderroost et al., 2017), que possui capacidade de verificar características, como o frescor de um alimento; etiquetas NFC com nanotubos de carbono, que informam a qualidade a partir da captação de gases e a interação com o aplicativo do smartphone (Wang et al., 2008); sensores comestíveis e etiquetas com leitor óptico, que analisam a presença de compostos provenientes da decomposição dos alimentos (Neethirajan \& Jayas, 2011), e sensores comestíveis compostos de ouro e seda, que permitem a identificação do frescor dos alimentos pela interação com aplicativos de smatphones (Tao et al., 2012), entre outros (Van Dorst et al., 2010).

O produto, objeto do presente estudo, foi definido com base em dispositivos que possuem a finalidade de reconhecimento de características dos produtos alimentícios. Trata-se de um dispositivo com a 
funcionalidade principal de reconhecimento instantâneo das características do alimento referentes à sua composição. Atualmente, estes dispositivos apresentam uma grande variedade de funcionalidades e características técnicas. Este estudo tem por objetivo identificar a melhor combinação desses atributos que deve ser incluída no dispositivo para identificar as características alimentares de alimentos customizados e a sua percepção de valor pelos clientes. A partir dos atributos considerados no estudo, foram desenvolvidas e testadas duas hipóteses de pesquisa considerando o efeito dos atributos e das variáveis moderadoras gênero e restrição alimentar na percepção de valor dos possíveis usuários.

\section{Material e métodos}

\subsection{Projeto experimental e análise conjunta}

Um projeto experimental representa uma sequência de experimentos a serem realizados (Montgomery, 2017). Sua aplicação na área alimentícia está relacionada à verificação da interação entre diferentes componentes que constituem o produto alimentício e as suas características (Zhou et al., 2008; Decloedt et al., 2016), e também para identificar a percepção dos consumidores perante diferentes combinações de atributos que compõem produtos alimentícios (Bruzzone et al., 2015).

Associada ao projeto experimental, pode ser utilizada a técnica de Análise Conjunta (AC). Esta técnica consiste em um método estabelecido e validado (Green \& Srinivasan, 1990; Gustafsson et al., 2013), que busca entender o comportamento do consumidor diante de atributos e estímulos (Claret et al., 2012). Para a presente pesquisa, por meio dos atributos considerados para o dispositivo, foi realizado um projeto experimental fatorial fracionado $2^{5-1}$ (Montgomery, 2017) com perfil completo (Green \& Srinivasan, 1978). Cada um dos atributos selecionados é detalhado a seguir.

\subsubsection{Portabilidade do dispositivo (A)}

A portabilidade consiste em um importante fator que apresenta efeito nos consumidores em sua decisão de escolha dos dispositivos tecnológicos (Kim, 2005). Os dispositivos portáteis de consumo devem ser compactos o suficiente para serem carregados no bolso ou na carteira dos usuários (Bortolin et al., 2006). Por meio da combinação de portabilidade dos celulares com a combinação de redes e computação, os smartphones tornaram-se uma tendência para incorporação de diversas funcionalidades (Guo et al., 2004). A capacidade dos smartphones em agregar outros serviços além da telecomunicação fornece versatilidade de situações de uso e estes se tornam integrados ao cotidiano dos usuários (Soikkeli et al., 2011). Essa versatilidade pode proporcionar uma necessidade no consumidor de ter várias funcionalidades reunidas em um único dispositivo. Entretanto, a miniaturização dos componentes também resulta em perda de desempenho do dispositivo.

\subsubsection{Precisão de medida (B)}

Ingredientes alimentares são fatores causadores de alergias alimentares e intolerâncias (Taylor \& Hefle, 2001). O desenvolvimento de regulamentações de rotulagem para pessoas com alergias alimentares é complexo, devido à variedade de formas com que os alimentos alergênicos e seus derivados são utilizados como ingredientes (Gendel, 2012). Para a personalização de alimentos, ainda se verifica uma dificuldade para o desenvolvimento de tabelas nutricionais personalizadas devido às inúmeras e possíveis combinações dos ingredientes. Além de prejudicar as pessoas que buscam uma alimentação mais saudável, a apresentação de informações nutricionais imprecisas pode resultar em riscos para consumidores que possuem dietas restritivas (Feldman et al., 2015). 


\subsubsection{Personalização de dieta (C)}

A variação genética nem sempre afeta diretamente o risco individual de doenças, mas algumas vezes este potencial em adquirir doenças pode ser explicado por certas condições alimentares (Tucker-Drob et al., 2013). Estudos nutrigenômicos buscam entender as diferentes necessidades dietéticas dos indivíduos a partir de suas características genéticas (German et al., 2011). A Nutrigenômica é uma ciência emergente na área de manutenção e promoção da saúde, associada a produtos e serviços alimentícios personalizados (Ghosh, 2010). Para reconhecimento de características genéticas, já existem métodos para análise das características do sangue do indivíduo por meio da espectrometria (Feng et al., 2010). De forma prática, no mercado podem ser identificados equipamentos com a mesma funcionalidade, tais como o Cor Kit (New Atlas, 2019), que propõe resultados imediatos para indicadores de saúde a partir da análise do sangue por espectrometria. Assim, a praticidade e a eficiência na análise sanguínea, em conjunto com o desenvolvimento de pesquisas na área de genômica nutricional, podem possibilitar uma personalização nutricional específica para cada perfil de usuário.

\subsubsection{Análise da qualidade do alimento (D)}

Qualidade de alimentos pode ser definida de acordo com as características físicas do produto alimentício ou por meio da percepção do consumidor (Grunert, 2005). A segurança de alimentos é uma prioridade desejável pelos consumidores e faz parte da qualidade de um produto alimentício (Van Rijswijk \& Frewer, 2008). Análises de qualidade são realizadas para reconhecimento da segurança dos alimentos quanto à identificação de contaminação microbiana, física e química, e de resíduos alergênicos (Müller \& Steinhart, 2007), que podem ser prejudiciais para consumidores com organismos deficientes. Dentre os métodos analíticos físico-químicos, pesquisas sobre a utilização de espectrometria podem ser encontradas na literatura com a finalidade de verificar a qualidade do alimento (Cen \& He, 2007; Hajslova et al., 2011). A utilização desse método é recomendada como uma eficiente ferramenta de análise da qualidade dos alimentos sem destruí-los ou modificá-los física e quimicamente (Gowen et al., 2007).

\subsubsection{Preço (E)}

$\mathrm{O}$ atributo (E) preço apresenta-se importante para compreender o comportamento do consumidor perante sua decisão de compra (Andrade et al., 2016; Asioli et al., 2016). A utilização da análise conjunta (AC) direcionada ao estudo de percepção de valor do consumidor perante características de produtos alimentícios, muitas vezes não considera o preço para montagem dos cenários que compõem os choice sets do experimento (Hoppert et al., 2014; Ares et al., 2014; Pelsmaeker et al., 2017). Como forma de complementar a compreensão do comportamento do cliente perante produtos alimentícios a partir da consideração do preço como atributo, pode ser utilizada a técnica de estimação do Willing to Pay (Heide \& Olsen, 2017). No entanto, a alternativa mais frequente na literatura é a utilização de valores representando níveis do preço para composição dos cenários (Asioli et al., 2016; Andrade et al., 2016). A definição destes níveis pode ser realizada utilizando os valores máximo e mínimo encontrados no mercado (Annunziata \& Vecchio, 2013; Andrade et al., 2016), a utilização de intervalos de preço (Boesch, 2014; Donadini et al., 2016; Meyerding, 2016) ou ainda de valores médios desses intervalos de preço (Sorenson \& Bogue, 2007; Asioli et al., 2016).

Para compreender o efeito do preço na percepção de valor do dispositivo, o presente estudo também considera o preço como um atributo do produto, assim como diversos outros estudos reportados na literatura (Boesch, 2014; Donadini et al., 2016; Asioli et al., 2016; Andrade et al., 2016; Calegari et al., 2018). Para identificar os níveis de preço, foi realizado um levantamento dos dispositivos encontrados no mercado para esta finalidade, totalizando 20 dispositivos com finalidade similar no mercado. Para apresentação do preço como atributo nos cenários correspondentes, fez-se necessário observar a dependência que esse atributo possui em relação aos demais atributos considerados por este estudo. Dessa forma, estimou-se o preço do 
dispositivo por meio de uma regressão linear (OLS), com os valores reais dos dispositivos encontrados no mercado como variável dependente e os atributos (A) portabilidade, (B) precisão de medida, (C) personalização de dieta e (D) análise da qualidade do alimento, como variáveis independentes. $\mathrm{O}$ resultado apresentou somente o atributo (B) precisão de medida com efeito significativo ( $p$-valor $<0,05$ ) sobre o (E) preço do dispositivo. Desta forma, a montagem dos cenários considerou os valores para o preço (E) associados ao atributo precisão de medida (B). Assim, o planejamento fatorial fracionado aplicado $\left(2^{5-1}\right)$ considerou a variável preço (E) aninhada à variável precisão de medida (B). Os valores superior e inferior da variável preço (E) foram estabelecidos por meio do intervalo de confiança obtido na regressão que testou sua dependência dos demais atributos avaliados no estudo, conforme apresentado na Tabela 1. Mesmo que diversos estudos de $\mathrm{AC}$ no setor alimentício considerem o preço como um atributo independente dos demais (Annunziata \& Vecchio, 2013; Meyerding, 2016; Asioli et al., 2016), esta característica pode resultar em cenários irreais, com preços altos para produtos de baixo valor em razão da sua associação com algum dos demais atributos (Hair et al., 2010). Para evitar este problema, foi considerado o preço aninhado de outro atributo para estruturação dos cenários experimentais, da mesma forma como reportado em outros estudos (Andrade et al., 2016).

\subsection{Desenvolvimento das hipóteses}

\subsubsection{Efeito das variáveis principais}

Para o presente estudo, consideraram-se como variáveis principais os atributos (A) portabilidade, (B) precisão de medida, (C) personalização de dieta, (D) análise de qualidade e (E) preço. Com base na descrição das variáveis principais, verifica-se a possibilidade de os consumidores estarem mais dispostos a escolher dispositivos portáteis, com uma maior acurácia, e que possuam as funcionalidades de personalização de dieta e de análise de qualidade do alimento, além de mais baratos. Dessa forma, propõe-se a primeira hipótese de pesquisa. É esperado que todas as variáveis principais se apresentem como significativas para atribuição de valor pelos clientes, sendo assim:

$\mathbf{H}_{1}$ : As variáveis principais exercem efeito significativo no valor que os consumidores estão dispostos a pagar pelo dispositivo.

\subsubsection{Efeito das variáveis moderadoras}

Em diversos estudos, as variáveis moderadoras podem apresentar efeitos significativos sobre a função estudada (Baron \& Kenny, 1986). Na literatura, é usual a incorporação de variáveis sociodemográficas como moderadoras em modelos de análise conjunta (Morton et al., 2012; Asioli et al., 2016). O presente estudo pretende compreender se diferentes perfis de consumidores moderam o efeito das variáveis principais sobre a disposição dos clientes a pagar pelo dispositivo estudado. Para isso, considerou-se o efeito das interações entre as variáveis principais e as variáveis moderadoras $(F)$ gênero e $(\mathrm{G})$ restrição (referente às pessoas que apresentam uma dieta restritiva).

Por meio da literatura, observa-se que mulheres usam serviços de conteúdo de informações para expressar sua singularidade, enquanto homens usufruem do mesmo serviço devido à sua funcionalidade (Hong et al., 2006). Pessoas do gênero feminino possuem maior preocupação quanto ao controle de peso (Wardle et al., 2004), quanto à sua imagem corporal e são mais propensas a critérios de escolha de alimentos que mantenham suas dietas (Beardsworth et al., 2002). Dessa forma, é esperado que mulheres sejam propensas a pagar mais por dispositivos que auxiliem sua dieta, a partir da personalização, da análise de qualidade e da precisão de medida. Para a variável moderadora $(\mathrm{G})$ restrição, destaca-se principalmente sua interação com a variável principal (B) precisão de medida. Os consumidores portadores de alergias alimentares tornam-se atentos às informações presentes nos rótulos e a precisão dessas declarações de ingredientes é fundamental para suas 
dietas específicas (Hefle et al., 2007). Dessa forma, é esperado uma maior disponibilidade a pagar por clientes que apresentem este tipo de alergia. Assim, propõe-se a segunda hipótese de pesquisa:

H2: A moderação das variáveis Gênero e Restrição sobre as variáveis principais promove efeito significativo no valor que os consumidores estão dispostos a pagar pelo dispositivo estudado.

\subsection{Planejamento dos cenários}

A partir da definição dos atributos, torna-se possível a modelagem da AC. A Tabela 1 apresenta os atributos considerados para esta pesquisa e seus respectivos níveis.

Tabela 1. Atributos e seus respectivos níveis.

\begin{tabular}{|c|c|}
\hline Atributos & Níveis dos atributos para a modelagem de análise conjunta \\
\hline \multirow{2}{*}{ Portabilidade } & Micromedidor embutido no celular (-) \\
\hline & Celular mais aparelho externo de medição $(+)$ \\
\hline \multirow{2}{*}{ Precisão de medida } & Alta precisão de medida/reconhecimento molecular $(+)$ \\
\hline & Baixa precisão de medida/baseado em composições padrões cadastrados (-) \\
\hline \multirow{2}{*}{ Personalização de dieta } & Dietas personalizadas, específicas para cada indivíduo $(+)$ \\
\hline & Dietas padronizadas para os indivíduos (-) \\
\hline \multirow{2}{*}{$\begin{array}{l}\text { Análise da qualidade do } \\
\text { alimento }\end{array}$} & Presença do identificador de qualidade de consumo \\
\hline & Ausência do identificador de qualidade de consumo (-) \\
\hline \multirow{2}{*}{ Preço* } & Alto (-) \\
\hline & Baixo $(+)$ \\
\hline
\end{tabular}

*Para montagem dos cenários, os níveis do preço são dependentes de cada nível de precisão: Para alta precisão, Preço nível alto $(\$ 378,75)$ e Preço nível baixo (\$277,71). Para baixa precisão, \$255,98 - Preço nível alto e \$124,94 - Preço nível baixo.

Este estudo ainda considerou a blocagem de cenários (choice sets) para que fosse possível a visualização de atributos que variam continuamente em uma região (Louviere et al., 2000) e para facilitar a decisão dos respondentes. Para o cenário escolhido, aplicou-se ainda o método de Willingness to Pay (WTP), usualmente utilizado pela literatura ( $\mathrm{Lu}$ et al., 2016; Wlömert \& Eggers, 2016), para compreender quanto os consumidores estão dispostos a pagar pelo dispositivo. A utilização desta forma de avaliação torna possível a mensuração métrica da variável dependente. Um exemplo do cenário para escolha do cliente e a mensuração de sua disponibilidade a pagar é apresentado na Figura 1. O instrumento de pesquisa utilizado pode ser acessado em sua forma completa em Calegari (2019). 
Uma análise conjunta para identificação dos atributos de um dispositivo para reconhecimento de características de produtos alimentícios customizados

Calegari, L. P., \& Fettermann, D. C.

\section{QUESTĀO 5: Qual dos cenários abaixo seria o produto que você mais gostaria de comprar?}
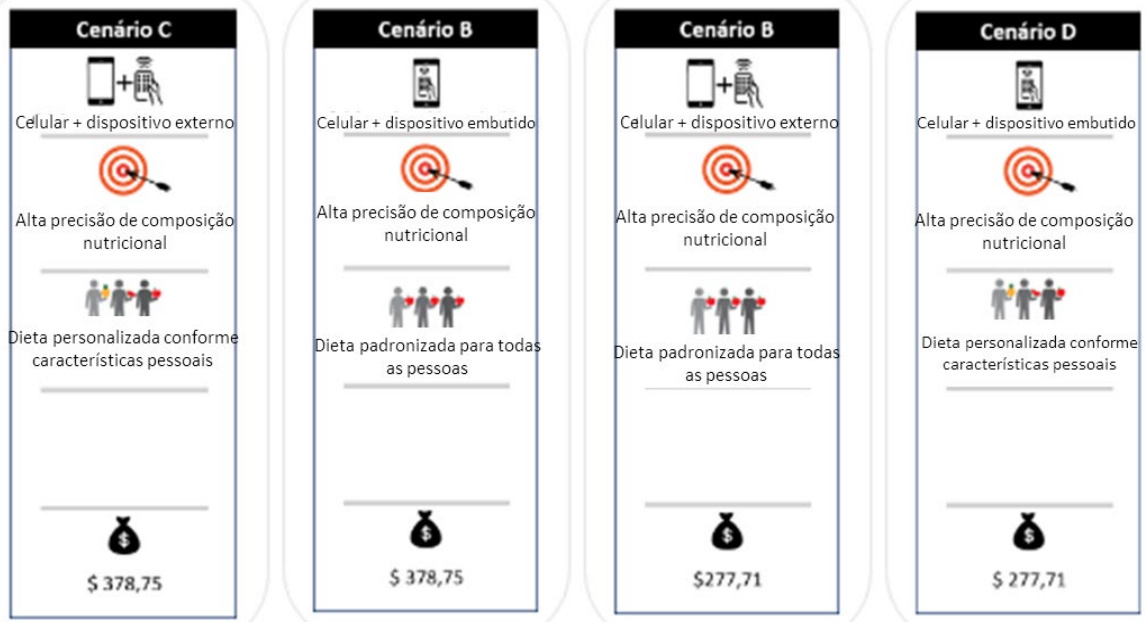

Em uma nota de 0 a 100, qual a seria o seu desejo de compra do cenário escolhido acima?

$\begin{array}{lllllllllll}0 & 10 & 20 & 30 & 40 & 50 & 60 & 70 & 80 & 90 & 100 \\ \text { Desejo de Compra } & & & & & & & & & & \end{array}$

Quanto você estaria disposto a pagar pelo produto descrito por esse cenário?

Apenas números, com os centavos separados por ", " (ex: 1,99)

Figura 1. Representação do quinto choice set.

\subsection{Universo amostral e coleta de dados}

A coleta de dados ocorreu entre os dias 13 de maio e 13 de junho de 2017, com respondentes de nacionalidade brasileira. Por meio de uma survey, foi realizada aplicação de um questionário online com auxílio da plataforma Qualtrics $^{\circledR}$. Foram obtidas 303 respostas completas e consideradas válidas para o experimento. O perfil dos respondentes (Tabela 2) indica um equilíbrio entre os gêneros, além de uma quantidade significativa de respondentes que possuem alguma restrição alimentar.

Tabela 2. Descritivo da amostra.

\begin{tabular}{cc}
\hline & Total de respondentes $(\mathbf{n}=\mathbf{3 0 3})$ \\
\hline Gênero & \\
Masculino & $48,51 \%$ \\
Feminino & $51,49 \%$ \\
Quanto a dieta & $31,35 \%$ \\
Dieta restritiva & $68,64 \%$ \\
\hline Nenhuma restrição & \\
\hline
\end{tabular}




\subsection{Análise dos dados}

Análise de Variância (ANOVA) é utilizada para a estimação de parâmetros dos modelos que explicam uma função (Platt \& Platt, 2002) e pode ser extensivamente encontrada na literatura de modelos de Análise Conjunta (Heide \& Olsen, 2017), inclusive em estudos sobre percepção de valor dos consumidores perante produtos alimentícios (Andrade et al., 2016; Asioli et al., 2016; Pelsmaeker et al., 2017). Para análise dos dados obtidos a partir da técnica de HIT-CBC (Two Level Choice Based Conjoint) (Eggers \& Sattler, 2009; Li \& Hudson, 2016; Kraus et al., 2016), foi realizada uma Análise de Variância aninhada (Montgomery, 2017), sendo considerada a variável WTP como dependente.

Desta forma, segundo modelo proposto por Næs et al. (2010) e utilizado por Asioli et al. (2016) e Hainmueller \& Hopkins (2015), na especificação do modelo GLM (Generalized Linear Model) para o presente estudo, consideraram-se os efeitos dos atributos principais e as interações entre os efeitos principais e as variáveis moderadoras (Figura 2). Os efeitos principais das variáveis moderadoras e as interações entre os efeitos principais das variáveis moderadoras não foram incluídos, em razão do modelo apenas diferenciar os efeitos relativos dos fatores comuns e não o nível geral de gostos dos consumidores (Asioli et al., 2016). O modelo de utilidade para o dispositivo tecnológico estudado $j$ para indivíduo $i$ na ocasião de escolha $t$ está representado na Equação 1. O modelo GLM foi estimado a partir do software Minitab ${ }^{\circledR}$.

$$
\begin{aligned}
& U_{i j t}=\beta_{1 i}(A)_{i j t}+\beta_{2 i}(B)_{i j t}+\beta_{3 i}(C)_{i j t}+\beta_{4 i}(D)_{i j t}+\beta_{5 i}(E(B))_{i j t}+\beta_{6 i}\left(A^{*} \text { Gênero }\right)_{i j t}+ \\
& \beta_{7 i}\left(A^{*} \text { Restrição }\right)_{i j t}+\mathrm{B}_{8 i}\left(B^{*} \text { Gênero }\right)_{i j t}++\beta_{9 i}\left(B^{*} \text { Restrição }\right)_{i j t}+\beta_{10 i}\left(C^{*} \text { Gênero }\right)_{i j t}+ \\
& \beta_{11 i}\left(C^{*} \text { Restrição }\right)_{i j t}+\beta_{12 i}\left(D^{*} \text { Gênero }\right)_{i j t}+\beta_{13 i}\left(D^{*} \text { Restrição }\right)_{i j t}+\beta_{14 i}\left(E(B)^{*} \text { Gênero }\right)_{i j t+}+ \\
& \beta_{15 i}(E(B) * \text { Restrição })_{i j t} \varepsilon_{i j}
\end{aligned}
$$

A partir das hipóteses formuladas e do modelo proposto, é apresentada de forma gráfica a estrutura de pesquisa utilizada neste trabalho (Figura 2).

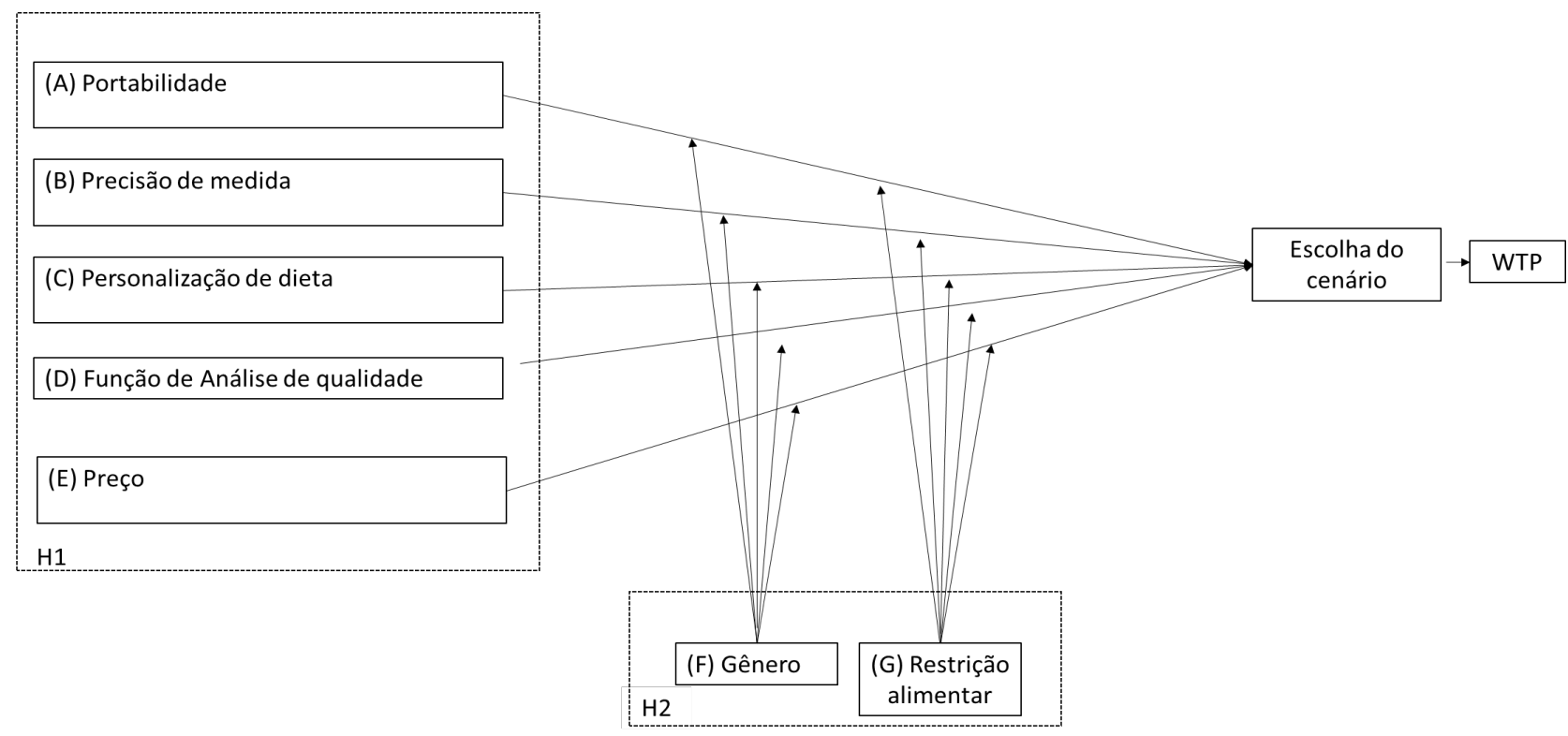

Figura 2. Estrutura de análise proposta para o estudo. 


\section{Resultados e discussões}

\subsection{Estimação dos parâmetros}

Primeiramente, foi verificada a suposição de aderência dos dados da variável dependente à distribuição normal (Montgomery, 2017). Em razão da rejeição da hipótese nula de aderência dos dados à distribuição normal (Anderson Darling AD: 0,499, $P$ valor $=0,208$ ), foi realizada uma transformação dos dados. Uma alternativa para atingir este objetivo é a realização da transformação de Box Cox (Box \& Cox, 1964). Esta transformação busca identificar o valor de $\lambda$ que maximiza a aderência dos dados à distribuição normal, viabilizando a utilização de técnica de análise proposta. Após a transformação dos dados, foi estimado o modelo de ANOVA aninhada (Equação 1) por meio do software estatístico Minitab ${ }^{\circledR}$, v.17. Para a análise de adequação do modelo proposto, foram seguidas as verificações de homogeneidade de variância, normalidade e aleatoriedade dos resíduos recomendadas por Hair et al. (2010). A análise gráfica dos resíduos do modelo indicou homogeneidade de variância e aleatoriedade dos resíduos. Além disso, a análise de normalidade dos resíduos não indicou indícios suficientes para rejeitar a hipótese de aderência dos mesmos à distribuição normal (Figura 3). Não foram identificadas evidências para rejeitar a validade do modelo proposto.

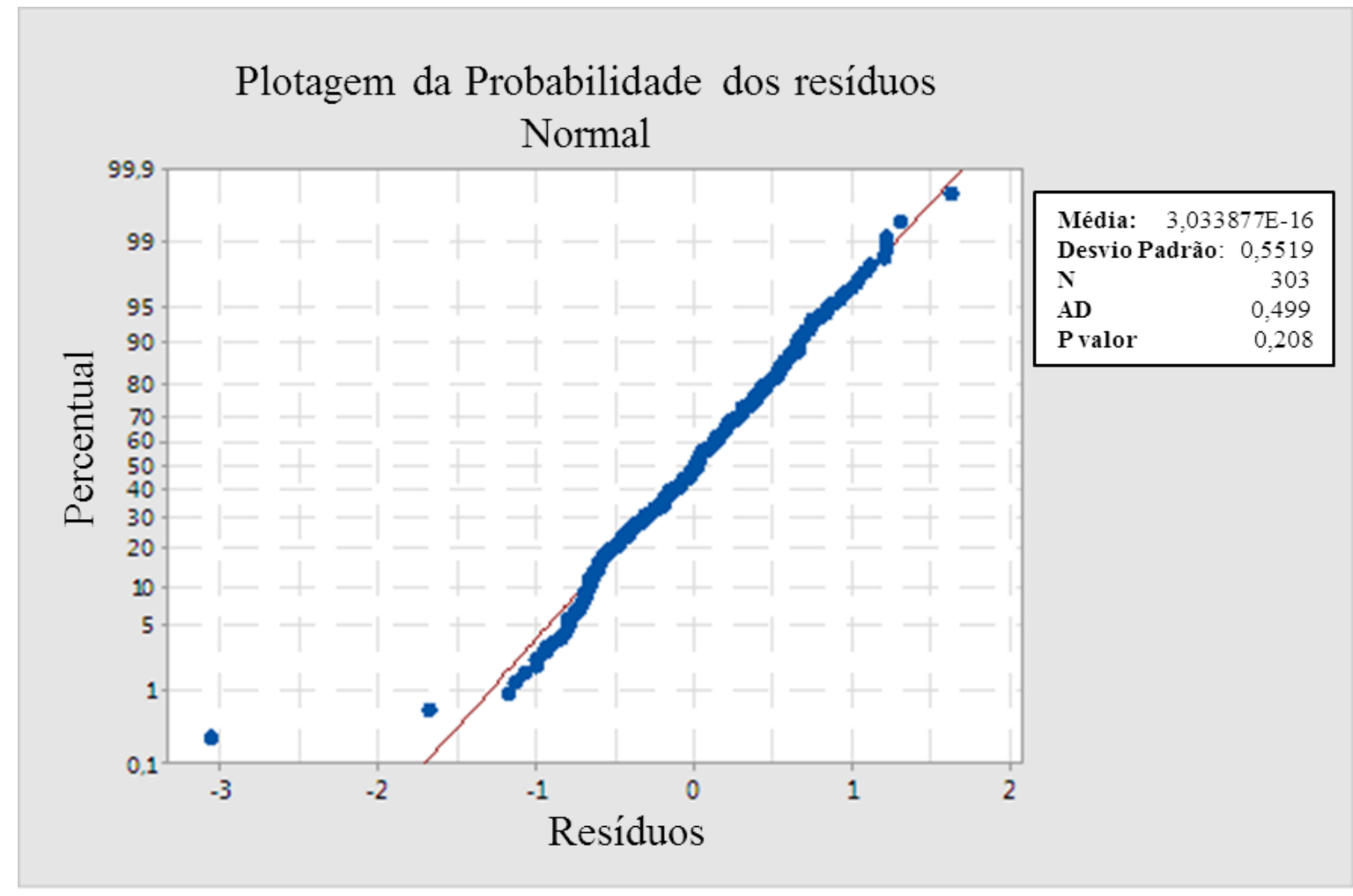

Figura 3. Gráfico dos resíduos padronizados.

A Tabela 3 apresenta os parâmetros estimados do modelo proposto para os efeitos principais dos fatores estudados. A partir dos resultados obtidos pela ANOVA Aninhada, foi possível observar quais os atributos do dispositivo verificados nesta pesquisa são significativos $(p$-valor $<0,05)$ na disponibilidade dos consumidores em pagar pelo dispositivo. $\mathrm{O}$ modelo proposto não apresentou indícios de falta de ajuste para descrever os dados experimentais coletados (lack-of-fit $>0,05$ ). 
Tabela 3. Estimação dos parâmetros do modelo e sua significância.

\begin{tabular}{|c|c|c|c|c|c|}
\hline Variável & Soma dos Quadrados & GL & Média dos Quadrados & Estatística $\mathbf{F}$ & p-valor \\
\hline \multicolumn{6}{|l|}{ Variáveis principais } \\
\hline A & 15825 & 1 & 15852 & 1,51 & 0,22 \\
\hline $\mathrm{B}$ & 106608 & 1 & 106608 & 11,6 & $0,006^{* *}$ \\
\hline $\mathrm{C}$ & 64 & 1 & 64 & 0,01 & 0,938 \\
\hline $\mathrm{D}$ & 45010 & 1 & 45010 & 4,3 & $0,039 * *$ \\
\hline $\mathrm{E}(\mathrm{B})$ & 15703 & 2 & 7851 & 0,87 & 0,699 \\
\hline \multicolumn{6}{|c|}{ Interação entre as variáveis principais e as variáveis moderadoras } \\
\hline A*Gênero & 6705 & 1 & 6705 & 0,64 & 0,424 \\
\hline A*Restrição & 4911 & 1 & 4911 & 0,47 & 0,494 \\
\hline B*Gênero & 105 & 1 & 105 & 0,01 & 0,909 \\
\hline B*Restrição & 150 & 1 & 150 & 0,01 & 0,912 \\
\hline C*Gênero & 8600 & 1 & 8600 & 0,82 & 0,366 \\
\hline C*Restrição & 14158 & 1 & 14158 & 1,35 & 0,246 \\
\hline D*Gênero & 19439 & 1 & 19439 & 1,86 & 0,174 \\
\hline D*Restrição & 54208 & 1 & 54208 & 5,18 & $0,024^{*}$ \\
\hline E*Gênero(B) & 4581 & 1 & 2290 & 0,22 & 0,804 \\
\hline E*Restrição(B) & 32868 & 1 & 16434 & 1,57 & 0,21 \\
\hline Erro & & 2952612 & 10470 & & \\
\hline Falta de Ajuste & 215772 & 23 & 9381 & 0,89 & 0,615 \\
\hline Erro Puro & & 2736839 & 10567 & & \\
\hline Total & 3555250 & 30 & & & \\
\hline
\end{tabular}

\section{2 $\mathrm{H}_{1}$ - Efeito das variáveis principais}

A partir da análise dos resultados obtidos para o efeito das variáveis principais, pode-se verificar que a hipótese $\mathrm{H}_{1}$ proposta pelo presente estudo foi parcialmente confirmada. Foi observado que apenas os atributos (B) precisão de medida e (D) análise de qualidade do alimento obtiveram significância a $5 \%$ ( $p$-valor $<0,05)$. Ainda vale destacar que o valor resultante para o atributo de precisão de medida torna-o o único atributo significativo a $1 \%$. Desta forma, a precisão de medida foi o atributo que mais influenciou na nota fornecida pelos consumidores.

Os resultados obtidos indicaram que os consumidores estão dispostos a pagar mais por dispositivos que possuam maior precisão de medida (B) das características alimentares e que sejam capazes de analisar a qualidade do produto alimentício (D). Como a presença dos demais atributos resultou não significativa no nível de 5\%, a percepção de valor dos consumidores apresenta-se indiferente quanto à portabilidade, função de personalização de dieta e preço.

\section{3 $\mathrm{H}_{2}$ - Efeito das interações das variáveis principais com as variáveis moderadoras}

A hipótese $\mathrm{H}_{2}$ também foi parcialmente aceita. A partir da análise dos efeitos das interações entre variáveis principais e variáveis moderadoras, verificou-se que somente a interação entre (D) análise de qualidade de consumo e (G) restrição influenciou o comportamento de escolha dos consumidores. Por meio da Figura 4, é possível observar que os respondentes que possuíam alguma restrição alimentar estavam propensos a pagar mais por dispositivos que apresentassem a função de análise de qualidade, enquanto que os respondentes que declarararam não dispor de restrições alimentares atribuíram valores inferiores para dispositivos que apresentavam tal função. Isto pode ser explicado pelo fato de que pessoas com dietas restritivas necessitam de maior confiabilidade sobre o produto que consomem (Hefle et al., 2007). A função de análise de qualidade pode ter valor para pessoas com dietas restritivas, à medida que fornecem informações úteis para que este laço de confiabilidade entre empresa e consumidor possa ser criado. As interações das variáveis principais com a variável gênero não 
foram significativas. Este resultado apresenta indícios de que mulheres e homens não diferem quanto aos valores que estão dispostos a pagar pelos atributos estudados, rejeitando parcialmente $\mathrm{H}_{2}$.

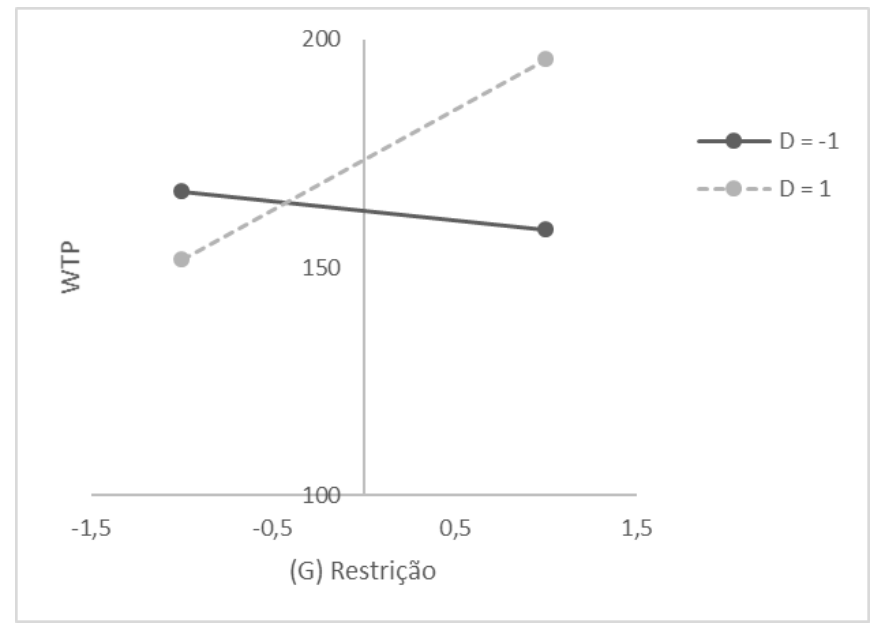

Figura 4. Interação entre Análise de qualidade de consumo e Restrição alimentar.

\section{Conclusão}

A survey foi respondida por um total de 361 pessoas, mas como $26,17 \%$ dos respondentes apresentaram respostas incompletas, foram consideradas válidas 303 respostas para análise. Entre estes respondentes, 91,08\% manifestaram interesse na aquisição do dispositivo estudado, confirmando a relevância do estudo.

Os resultados obtidos indicam que o dispositivo deve apresentar pelo menos precisão de medida-B e análise de qualidade-D. Os resultados também indicam uma valorização do atributo análise de qualidade perante os clientes com restrição alimentar.

O método de Análise Conjunta apresenta-se como uma estratégia de estruturação para realização de testes estatísticos a partir de experimentos de preferência no mercado consumidor. Os resultados obtidos a partir da $\mathrm{AC}$ ainda podem servir como base a direcionamentos quanto ao investimento em pesquisas que possuam a finalidade de desenvolver tais dispositivos. Dessa forma, como contribuições teóricas, por meio do dispositivo tecnológico estudado, torna-se possível a acessibilidade imediata a vários formatos de etiquetas nutricionais para o produto alimentício (Volkova \& Ni Mhurchu, 2015). Desta forma, pode ser considerado um facilitador para customização em massa dos alimentos, pela capacidade de gerar informações para desenvolvimento de rótulos personalizados para cada produto alimentício. Com isso, surge também a oportunidade da personalização de dietas específicas, que possibilitariam avanços na direção de estudos nutrigenômicos (German et al., 2011).

A utilização de big data analytics e inteligência artificial permite superar a dificuldade das empresas em compreender o comportamento dos mercados consumidores (Tortorella et al., 2015; Echeveste et al., 2017; Fettermann et al., 2018), a partir do monitoramento online do comportamento do usuário. Também permite a identificação de oportunidades de novos produtos, serviços e a melhor compreensão do processo de uso deste tipo de dispositivo pelos clientes. A utilização dos dados de utilização deste tipo de dispositivo ainda pode direcionar a construção de tendências de consumo e o desenvolvimento de novas pesquisas e produtos alimentícios.

Em relação às limitações deste estudo, é importante mencionar que a abrangência amostral, quanto à localização dos respondentes, possuiu uma superioridade de pessoas localizadas nas regiões Sul e CentroOeste do Brasil. Estudos futuros poderiam ser replicados para comparar percepções de consumidores com origem em diferentes países. Apesar de a amostra representar o estrato de pessoas com restrições, outra 
limitação do presente estudo está relacionada à dispersão amostral para o desenvolvimento de produtos focados em perfis específicos da população. Desta forma, experimentos futuros poderiam ter população amostral formada exclusivamente por pessoas que possuem algum tipo de restrição.

Pesquisas futuras também poderiam ser desenvolvidas para aprimorar os dispositivos já existentes, conforme os desejos dos consumidores resultantes para o presente estudo. Ainda poderiam abordar o desenvolvimento de uma rede de comunicação entre o dispositivo, o consumidor e a empresa. Isto possibilitaria a integração do consumidor ao processo de produção, a partir da facilitação da identificação de suas necessidades.

\section{Referências}

Andrade, J. C., Nalério, É. S., Giongo, C., De Barcellos, M. D., Ares, G., \& Deliza, R. (2016). Influence of evoked contexts on rating-based conjoint analysis: Case study with lamb meat. Food Quality and Preference, 53, 168-175. http://dx.doi.org/10.1016/j.foodqual.2016.06.013

Annunziata, A., \& Vecchio, R. (2013). Consumer perception of functional foods: A conjoint analysis with probiotics. Food Quality and Preference, 28(1), 348-355. http://dx.doi.org/10.1016/j.foodqual.2012.10.009

Arens-Volland, A. G., Spassova, L., \& Bohn, T. (2015). Promising approaches of computer-supported dietary assessment and management: Current research status and available applications. International Journal of Medical Informatics, 84(12), 997-1008. PMid:26321486. http://dx.doi.org/10.1016/j.jjmedinf.2015.08.006

Ares, G., Mawad, F., Giménez, A., \& Maiche, A. (2014). Influence of rational and intuitive thinking styles on food choice: Preliminary evidence from an eye-tracking study with yogurt labels. Food Quality and Preference, 31, 28-37. http://dx.doi.org/10.1016/j.foodqual.2013.07.005

Asioli, D., Næs, T., Øvrum, A., \& Almli, V. L. (2016). Comparison of rating-based and choice-based conjoint analysis models: A case study based on preferences for iced coffee in Norway. Food Quality and Preference, 48, 174-184. http://dx.doi.org/10.1016/j.foodqual.2015.09.007

Balcombe, K., Fraser, I., Lowe, B., \& Monteiro, D. S. (2016). Information customization and food choice. American Journal of Agricultural Economics, 98(1), 54-73. http://dx.doi.org/10.1093/ajae/aav033

Baron, R. M., \& Kenny, D. A. (1986). The moderator-mediator variable distinction in social psychological research: Conceptual, strategic, and statistical considerations. Journal of Personality and Social Psychology, 51(6), 1173-1182. PMid:3806354. http://dx.doi.org/10.1037/0022-3514.51.6.1173

Beardsworth, A., Bryman, A., Keil, T., Goode, J., Haslam, C., \& Lancashire, E. (2002). Women, men and food: The significance of gender for nutritional attitudes and choices. British Food Journal, 104(7), 470-491. http://dx.doi.org/10.1108/00070700210418767

Boesch, I. (2014). Processing companies' preferences for attributes of beef in Switzerland. Meat Science, 96(1), 153-156. PMid:23896149. http://dx.doi.org/10.1016/j.meatsci.2013.05.045

Bortolin, C., Nguyen, L., \& Redford, L. (2006). U.S. Patent No. 7,104,446. Washington, DC: U.S. Patent and Trademark Office.

Box, G. E. P., \& Cox, D. R. (1964). An analysis of transformations. Journal of the Royal Statistical Society. Series $B$. Methodological, 26(2), 211-243. http://dx.doi.org/10.1111/j.2517-6161.1964.tb00553.x

Bruzzone, F., Vidal, L., Antúnez, L., Giménez, A., Deliza, R., \& Ares, G. (2015). Comparison of intensity scales and CATA questions in new product development: Sensory characterisation and directions for product reformulation of milk desserts. Food Quality and Preference, 44, 183-193. http://dx.doi.org/10.1016/j.foodqual.2015.04.017

Burton, S., Howlett, E., \& Tangari, A. H. (2009). Food for thought: How will the nutrition labeling of quick service restaurant menu items influence consumers' product evaluations, purchase intentions, and choices? Journal of Retailing, 85(3), 258-273. http://dx.doi.org/10.1016/j.jretai.2009.04.007

Calegari, L. P. (2019). Questionário para dispositivo para reconhecimento de características de produtos alimentícios customizados. Recuperado em 16 de maio de 2019, de https://qtrial2016q3az1.qualtrics.com/jfe/form/SV_agBwhgz2ov8ZLpj

Calegari, L. P., Barbosa, J., Marodin, G. A., \& Fettermann, D. C. (2018). A conjoint analysis to consumer choice in Brazil: Defining device attributes for recognizing customized foods characteristics. Food Research International, 109, 1-13. PMid:29803431. http://dx.doi.org/10.1016/j.foodres.2018.03.080

Cen, H., \& He, Y. (2007). Theory and application of near infrared reflectance spectroscopy in determination of food quality. Trends in Food Science \& Technology, 18(2), 72-83. http://dx.doi.org/10.1016/j.tifs.2006.09.003

Chen, P., Liang, Y. H., \& Lin, T. C. (2014). Implementing a cooking and dietary management system using RFID technology. Mathematical Problems in Engineering, 2014, 1-10. http://dx.doi.org/10.1155/2014/234614

Claret, A., Guerrero, L., Aguirre, E., Rincón, L., Hernández, M. D., Martínez, I., Benito Peleteiro, J., Grau, A., \& RodríguezRodríguez, C. (2012). Consumer preferences for sea fish using conjoint analysis: Exploratory study of the importance of country of origin, obtaining method, storage conditions and purchasing price. Food Quality and Preference, 26(2), 259-266. http://dx.doi.org/10.1016/j.foodqual.2012.05.006 
Coskun, A. F., Wong, J., Khodadadi, D., Nagi, R., Tey, A., \& Ozcan, A. (2013). A personalized food allergen testing platform on a cellphone. Lab on a Chip, 13(4), 636-640. PMid:23254910. http://dx.doi.org/10.1039/C2LC41152K

Decloedt, A. I., Van Landschoot, A., \& Vanhaecke, L. (2016). Fractional factorial design-based optimisation and application of an extraction and UPLC-MS/MS detection method for the quantification of phytosterols in food, feed and beverages low in phytosterols. Analytical and Bioanalytical Chemistry, 408(27), 7731-7744. PMid:27565790. http://dx.doi.org/10.1007/s00216016-9870-8

Donadini, G., Fumi, M. D., Kordialik-Bogacka, E., Maggi, L., Lambri, M., \& Sckokai, P. (2016). Consumer interest in specialty beers in three European markets. Food Research International, 85, 301-314. PMid:29544848. http://dx.doi.org/10.1016/j.foodres.2016.04.029

Eggers, F., \& Sattler, H. (2009). Hybrid individualized two-level choice-based conjoint (HIT-CBC): A new method for measuring preference structures with many attribute levels. International Journal of Research in Marketing, 26(2), 108-118. http://dx.doi.org/10.1016/j.jiresmar.2009.01.002

Feldman, C., Murray, D., Chavarria, S., \& Zhao, H. (2015). Menu label accuracy at a university's foodservices: An exploratory recipe nutrition analysis. Appetite, 92, 24-28. PMid:25958116. http://dx.doi.org/10.1016/j.appet.2015.05.001

Feng, S., Chen, R., Lin, J., Pan, J., Chen, G., Li, Y., Cheng, M., Huang, Z., Chen, J., \& Zeng, H. (2010). Nasopharyngeal cancer detection based on blood plasma surface-enhanced Raman spectroscopy and multivariate analysis. Biosensors \& Bioelectronics, 25(11), 2414-2419. PMid:20427174. http://dx.doi.org/10.1016/j.bios.2010.03.033

Fettermann, D. C., Cavalcante, C. G. S., Almeida, T. D. D., \& Tortorella, G. L. (2018). How does Industry 4.0 contribute to operations management? Journal of Industrial and Production Engineering, 35(4), 255-268.

http://dx.doi.org/10.1080/21681015.2018.1462863

Fettermann, D., Echeveste, M. E. S., \& Tortorella, G. L. (2017). The benchmarking of the use of toolkit for mass customization in the automobile industry. Benchmarking, 24(6), 1767-1783. http://dx.doi.org/10.1108/BIJ-01-2016-0002

Gendel, S. M. (2012). Comparison of international food allergen labeling regulations. Regulatory Toxicology and Pharmacology, 63(2), 279-285. PMid:22565206. http://dx.doi.org/10.1016/j.yrtph.2012.04.007

German, J. B., Zivkovic, A. M., Dallas, D. C., \& Smilowitz, J. T. (2011). Nutrigenomics and personalized diets: What will they mean for food? Annual Review of Food Science and Technology, 2(1), 97-123. PMid:22129377.

http://dx.doi.org/10.1146/annurev.food.102308.124147

Ghosh, D. (2010). Personalised food: How personal is it? Genes \& Nutrition, 5(1), 51-53. PMid:19763657. http://dx.doi.org/10.1007/s12263-009-0139-0

Gowen, A., Odonnell, C., Cullen, P., Downey, G., \& Frias, J. (2007). Hyperspectral imaging: An emerging process analytical tool for food quality and safety control. Trends in Food Science \& Technology, 18(12), 590-598. http://dx.doi.org/10.1016/j.tifs.2007.06.001

Green, P. E., \& Srinivasan, V. (1978). Conjoint analysis in consumer research: Issues and outlook. The Journal of Consumer Research, 5(2), 103-123. http://dx.doi.org/10.1086/208721

Green, P. E., \& Srinivasan, V. (1990). Conjoint analysis in marketing: New developments with implications for research and practice. Journal of Marketing, 54(4), 3-19. http://dx.doi.org/10.1177/002224299005400402

Grunert, K. G. (2005). Food quality and safety: Consumer perception and demand. European Review of Agriculture Economics, 32(3), 369-391. http://dx.doi.org/10.1093/eurrag/jbi011

Guo, C., Wang, H. J., \& Zhu, W. (2004). Smart-phone attacks and defenses. In Proceedings of the 3rd Workshop on Hot Topics in Networks (HotNets III). San Diego.

Gustafsson, A., Herrmann, A., \& Huber, F. (Eds.). (2013). Conjoint measurement: Methods and applications. Berlin: Springer Science \& Business Media.

Hainmueller, J., \& Hopkins, D. J. (2015). The hidden American immigration consensus: A conjoint analysis of attitudes toward immigrants. American Journal of Political Science, 59(3), 529-548. http://dx.doi.org/10.1111/ajps.12138

Hair, J. F., Anderson, R. E., Babin, B. J., \& Black, W. C. (2010). Multivariate data analysis: A global perspective (Vol. 7). New Jersey: Pearson Upper Saddle River.

Hajslova, J., Cajka, T., \& Vaclavik, L. (2011). Challenging applications offered by direct analysis in real time (DART) in foodquality and safety analysis. Trends in Analytical Chemistry, 30(2), 204-218. http://dx.doi.org/10.1016/j.trac.2010.11.001

Hefle, S. L., Furlong, T. J., Niemann, L., Lemon-Mule, H., Sicherer, S., \& Taylor, S. L. (2007). Consumer attitudes and risks associated with packaged foods having advisory labeling regarding the presence of peanuts. The Journal of Allergy and Clinical Immunology, 120(1), 171-176. PMid:17544097. http://dx.doi.org/10.1016/j.jaci.2007.04.013

Heide, M., \& Olsen, S. O. (2017). Influence of packaging attributes on consumer evaluation of fresh cod. Food Quality and Preference, 60, 9-18. http://dx.doi.org/10.1016/j.foodqual.2017.02.015

Hong, S. J., Tam, K. Y., \& Kim, J. (2006). Mobile data service fuels the desire for uniqueness. Communications of the ACM 49(9), 89-94. http://dx.doi.org/10.1145/1151030.1151034

Hoppert, K., Mai, R., Zahn, S., Schwarz, P. E., Hoffmann, S., \& Rohm, H. (2014). Is there a fit in cognitive and sensory evaluation of yogurt? The moderating role of nutrition training. Food Quality and Preference, 31, 65-68.

http://dx.doi.org/10.1016/j.foodqual.2013.08.001

Kim, J. (2005). The effect of mobile number portability on switching costs in the telecommunication industry. Madison: Department of Economics, University of Wisconsin-Madison. 
Kraus, S., Meier, F., Eggers, F., Bouncken, R. B., \& Schuessler, F. (2016). Standardisation vs. adaption: A conjoint experiment on the influence of psychic, cultural and geographical distance on international marketing mix decisions. European Journal of International Management, 10(2), 127-156. http://dx.doi.org/10.1504/EJIM.2016.074468

Kriflik, L. S., \& Yeatman, H. (2005). Food scares and sustainability: A consumer perspective. Health Risk \& Society, 7(1), 11-24. http://dx.doi.org/10.1080/13698570500042439

Li, J., \& Hudson, S. (2016). Conjoint analysis of consumer preferences to destination brand attributes (Advancing Tourism Research Globally, No. 22). Massachusetts: Travel and Tourism Research Association.

Louviere, J. J., Hensher, D. A., \& Swait, J. D. (2000). Stated choice methods: Analysis and applications. New York: Cambridge University Press. http://dx.doi.org/10.1017/CBO9780511753831.

Lu, J., Wu, L., Wang, S., \& Xu, L. (2016). Consumer preference and demand for traceable food attributes. British Food Journal, 118(9), 2140-2156. http://dx.doi.org/10.1108/BFJ-12-2015-0461

Makarona, E., Petrou, P., Kakabakos, S., Misiakos, K., \& Raptis, I. (2016). Point-of-Need bioanalytics based on planar optical interferometry. Biotechnology Advances, 34(3), 209-233. PMid:26876018. http://dx.doi.org/10.1016/j.biotechadv.2016.02.005

Meyerding, S. (2016). GH. Consumer preferences for food labels on tomatoes in Germany: A comparison of a quasi-experiment and two stated preference approaches. Appetite, 103, 105-112. PMid:27037221. http://dx.doi.org/10.1016/j.appet.2016.03.025

Meyers, A., Johnston, N., Rathod, V., Korattikara, A., Gorban, A., Silberman, N., \& Murphy, K. P. (2015). Im2Calories: towards an automated mobile vision food diary. In Proceedings of the IEEE International Conference on Computer Vision (pp. 12331241). New York: IEEE.

Montgomery, D. C. (2017). Design and analysis of experiments. New York: John Wiley \& Sons.

Morton, R. L., Snelling, P., Webster, A. C., Rose, J., Masterson, R., Johnson, D. W., \& Howard, K. (2012). Factors influencing patient choice of dialysis versus conservative care to treat end-stage kidney disease. Canadian Medical Association Journal, 184(5), E277-E283. PMid:22311947. http://dx.doi.org/10.1503/cmaj.111355

Müller, A., \& Steinhart, H. (2007). Recent developments in instrumental analysis for food quality. Food Chemistry, 102(2), 436444. http://dx.doi.org/10.1016/j.foodchem.2006.10.015

Næs, T., Lengard, V., Bølling Johansen, S., \& Hersleth, M. (2010). Alternative methods for combining design variables and consumer preference with information about attitudes and demographics in conjoint analysis. Food Quality and Preference, 21(4), 368-378. http://dx.doi.org/10.1016/j.foodqual.2009.09.004

Neethirajan, S., \& Jayas, D. S. (2011). Nanotechnology for the food and bioprocessing industries. Food and Bioprocess Technology, 4(1), 39-47. http://dx.doi.org/10.1007/s11947-010-0328-2

New Atlas. (2019). Smartphone spectroscopy kit brings blood test info home. Recuperado em 16 de maio de 2019, de http://newatlas.com/cor-blood-chemistry-health-tracker/42608

Pelsmaeker, S., Schouteten, J. J., Lagast, S., Dewettinck, K., \& Gellynck, X. (2017). Is taste the key driver for consumer preference? A conjoint analysis study. Food Quality and Preference, 62, 323-331. http://dx.doi.org/10.1016/j.foodqual.2017.02.018

Platt, H. D., \& Platt, M. B. (2002). Predicting corporate financial distress: Reflections on choice-based sample bias. Journal of Economics and Finance, 26(2), 184-199. http://dx.doi.org/10.1007/BF02755985

Reitberger, W., Spreicer, W., \& Fitzpatrick, G. (2014). Situated and mobile displays for reflection on shopping and nutritional choices. Personal and Ubiquitous Computing, 18(7), 1721-1735. http://dx.doi.org/10.1007/s00779-014-0781-4

Schuldt, J. P. (2013). Does green mean healthy? Nutrition label color affects perceptions of healthfulness. Health Communication, 28(8), 814-821. PMid:23444895. http://dx.doi.org/10.1080/10410236.2012.725270

Soikkeli, T., Karikoski, J., \& Hammainen, H. (2011). Diversity and end user context in smartphone usage sessions. In Proceedings of the 5th International Conference on Next Generation Mobile Applications, Services and Technologies (NGMAST) (pp. 7-12). New York: IEEE. http://dx.doi.org/10.1109/NGMAST.2011.12.

Sorenson, D., \& Bogue, J. (2007). Concept optimisation in innovation through conjoint analysis: A market-oriented approach to designing new functional beverages. Journal of International Food \& Agribusiness Marketing, 19(2-3), 53-75. http://dx.doi.org/10.1300/J047v19n02_04

Tao, H., Brenckle, M. A., Yang, M., Zhang, J., Liu, M., Siebert, S. M., Averitt, R. D., Mannoor, M. S., McAlpine, M. C., Rogers, J. A., Kaplan, D. L., \& Omenetto, F. G. (2012). Silk-based conformal, adhesive, edible food sensors. Advanced Materials, 24(8), 1067-1072. PMid:22266768. http://dx.doi.org/10.1002/adma.201103814

Taylor, S. L., \& Hefle, S. L. (2001). Ingredient and labeling issues associated with allergenic foods. Allergy, 56(s67, Supl. 67), 64-69. PMid:11298013. http://dx.doi.org/10.1034/j.1398-9995.2001.00920.x

Tortorella, G. L., Fettermann, D. C., Marodin, G. A., \& Fogliatto, F. S. (2015). Lean product development (LPD) enablers for product development process improvement. In J. Paulo Davim (Ed.), Research advances in industrial engineering (pp. 31-57). Cham: Springer. http://dx.doi.org/10.1007/978-3-319-17825-7_3.

Tucker-Drob, E. M., Briley, D. A., \& Harden, K. (2013). Paige. Genetic and environmental influences on cognition across development and context. Current Directions in Psychological Science, 22(5), 349-355. PMid:24799770. http://dx.doi.org/10.1177/0963721413485087

Van Dorst, B., Mehta, J., Bekaert, K., Rouah-Martin, E., De Coen, W., Dubruel, P., Blust, R., \& Robbens, J. (2010). ROBBENS, J. Recent advances in recognition elements of food and environmental biosensors: A review. Biosensors \& Bioelectronics, 26(4), 1178-1194. PMid:20729060. http://dx.doi.org/10.1016/j.bios.2010.07.033 
Uma análise conjunta para identificação dos atributos de um dispositivo para reconhecimento de características de produtos alimentícios customizados

Calegari, L. P., \& Fettermann, D. C.

Van Rijswijk, W., \& Frewer, L. J. (2008). Consumer perceptions of food quality and safety and their relation to traceability. British Food Journal, 110(10), 1034-1046. http://dx.doi.org/10.1108/00070700810906642

Vanderroost, M., Ragaert, P., Verwaeren, J., De Meulenaer, B., De Baets, B., \& Devlieghere, F. (2017). The digitization of a food package's life cycle: Existing and emerging computer systems in the pre-logistics phase. Computers in Industry, 87, 1-14. http://dx.doi.org/10.1016/j.compind.2017.02.002

Vasiljevic, M., Pechey, R., \& Marteau, T. M. (2015). Making food labels social: The impact of colour of nutritional labels and injunctive norms on perceptions and choice of snack foods. Appetite, 91, 56-63. PMid:25841647. http://dx.doi.org/10.1016/j.appet.2015.03.034

Volkova, E., \& Ni Mhurchu, C. (2015). The influence of nutrition labeling and point-of-purchase information on food behaviours. Current Obesity Reports, 4(1), 19-29. PMid:26627087. http://dx.doi.org/10.1007/s13679-014-0135-6

Wang, F., Gu, H., \& Swager, T. M. (2008). Carbon nanotube/polythiophene chemiresistive sensors for chemical warfare agents. Journal of the American Chemical Society, 130(16), 5392-5393. PMid:18373343. http://dx.doi.org/10.1021/ja710795k

Wardle, J., Haase, A. M., Steptoe, A., Nillapun, M., Jonwutiwes, K., \& Bellisie, F. (2004). Gender differences in food choice: The contribution of health beliefs and dieting. Annals of Behavioral Medicine, 27(2), 107-116. PMid:15053018. http://dx.doi.org/10.1207/s15324796abm2702_5

Wlömert, N., \& Eggers, F. (2016). Predicting new service adoption with conjoint analysis: External validity of BDM-based incentive-aligned and dual-response choice designs. Marketing Letters, 27(1), 195-210. http://dx.doi.org/10.1007/s11002-0149326-x

Zhou, X. X., Pan, Y. J., Wang, Y. B., \& Li, W. F. (2008). Optimization of medium composition for nisin fermentation with response surface methodology. Journal of Food Science, 73(6), M245-M249. PMid:19241552. http://dx.doi.org/10.1111/j.17503841.2008.00836.x 